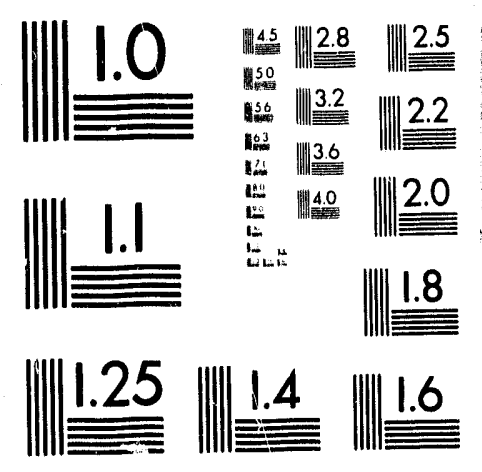



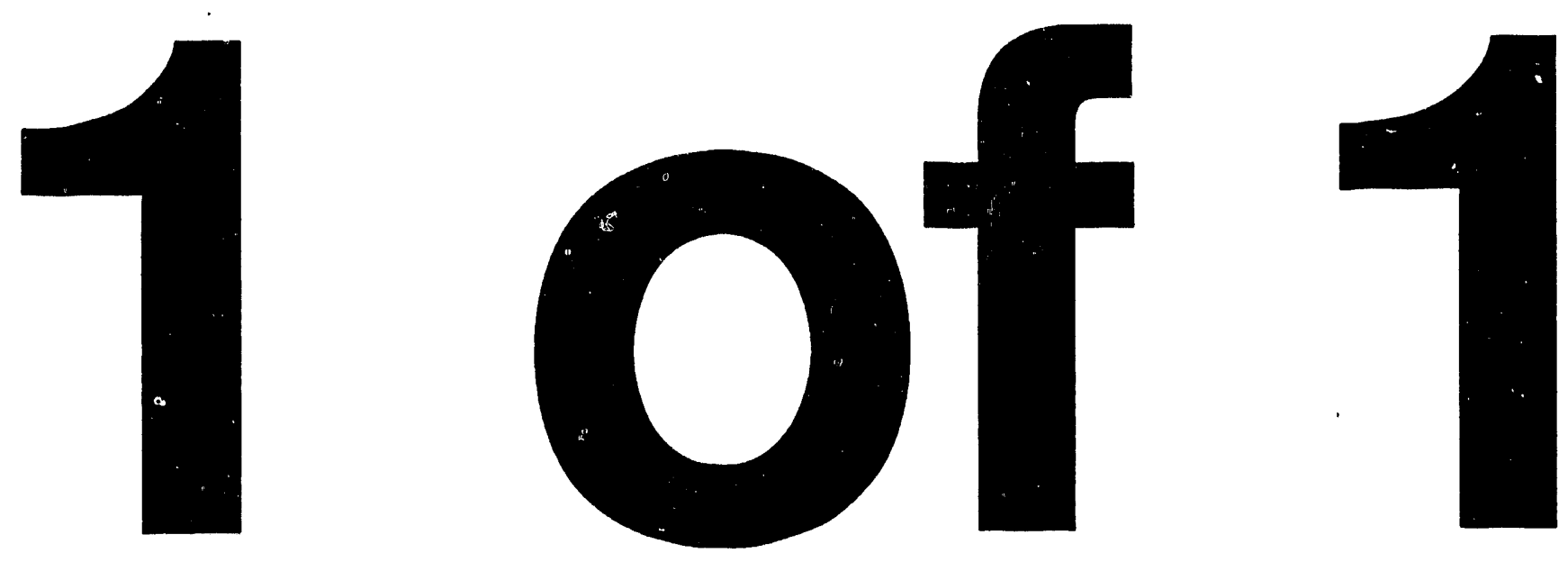
OCT 191803

$0 S T 1$

\section{Associated-Particle Sealed-Tube Neutron Probe For Characterization of MATERIALS}

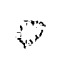

\author{
ED RHODES \\ Argonne National Laboratory, RE Building 208 \\ 9700 S. Cass Ave., Argonne, IL, USA 60439 \\ Phone 708/252-4575, Fax 708/252-4780
}

\section{Charles E. DiCKERMAN}

Argonne National Laboratory, RE Building $208 \cdot$ 9700 S. Cass Ave., Argonne, IL, USA 60439

Phone 708/252-4622, Fax 708/252-4780

Charles W. Peters

$5235 \mathrm{~N}$. Whispering Hills Lane, Tucson, AZ, USA 85704 Phone 602/292-1129, Fax 602/887-9108

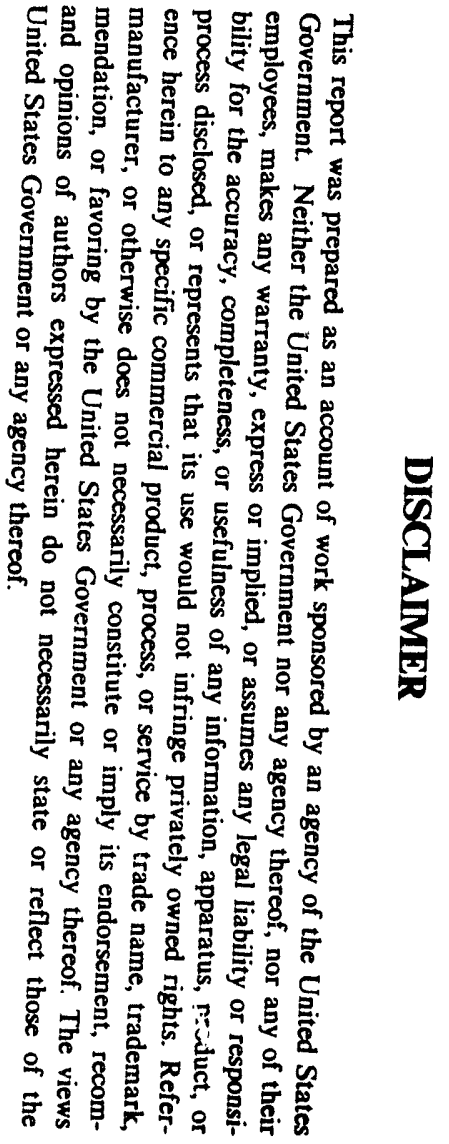

Submitted to Active Probe Technologies Conference (Chairs G. Harding and R. C. Lanza) of International Symposium on Substance Identification Technologies 4-8 October 1993, Innshruck, Austria 


\title{
Associated-particle sealed-tube neutron probe for characterization of materials*
}

\author{
E. Rhodes and C. E. Dickerman \\ Argonne National Laboratory, Reactor Engineering Division \\ 9700 S. Cass Ave., Argonne, Illinois, USA 6(1439 \\ C. W. Peters \\ $5235 \mathrm{~N}$. Whispering Hills Lane \\ Tucson, Arizona, USA 85704
}

\begin{abstract}
A relatively small and inexpensive neutron diagnostic probe system has been developed that can identify and image most elements having a larger atomic number than boron. It has the potential to satisfy van-mobile and fixed-portal requirements for nondestructive detection of contraband drugs, explosives, and nuclear and chemical warfare weapon materials, and for treaty verification of sealed munitions and remediation of radioactive waste. The probe is based on a unique non-pulsed associated-particle sealed-tube neutron generator (APSTNG) that inter-ngates the object of interest with a low-intensity beam of 14-MeV neutrons generated from the deuterium-tritium reaction and detects the alpha-particle associated with each neutron. Gamma-ray spectra of resulting neutron reactions (primarily inelastic scattering) identify nuclides associated with drugs, explosives, and other contraband. Flight times determined from detection times of the gamma-rays and alpha-particles yield a separate coarse tomographic image of each identified nuclide. Chemical substances are identified and imaged by comparing relative spectral line intensities with ratios of elements in reference compounds. The figh-energy neutrons and gamma-rays will penetrate large objects and dense materials. The source and emission detection systems can be located on the same side of the interrogated volume, allowing measurements with access to one side only. A relatively high signal-to-background ratio is obtained and maximum information is extracted from each detected gamma-ray, yet high-bandwidth data acquisition is not required. The APSTNG also forms the basis for a compact fast-neutron transmission imaging system. No collimators for the source or detectors are required, and only minimal personnel radiation shielding is needed. The small and relatively inexpensive neutron generator tube exhibits high reliability and can be quickly replaced. The detector arrays and associated electronics can be made reliable with low maintenance cost. Surveillance systems based on APSTNG technology can avoid the large physical size, high capital and operating expenses, and reliability problems of complex accelerators.
\end{abstract}

\section{INTRODUCTION}

Gamma-ray and neutron hodoscopes can be combined with a recently developed neutron diagnostic probe for a range of van-mobile and fixed-portal applications for NDA (nondestructive analysis), including detection of explosives and drugs in aviation, customs, and physical security environments, arms control treaty verification, nonproliferation surveillance of SNM (special nuclear material) and CW (chemical warfare) agents, and remediation of radwaste and pollutants. In the hodoscope concept, an array of radiation detectors is used to image or detect objects inside opaque containers. Argonne interest stems from the fast-neutron hodoscope ${ }^{1}$ in use at the Transient Reactor Test (TREAT) Facility for many years to image motion inside thick steel capsules of reactor fuel undergoing destructive testing that simulates hypothetical core-disruptive accidents.

\footnotetext{
"Argonne National Laboratory is owned by the U. S. government and operated by the University of Chicago under the provisions of a contract with the Department of Energy. This work was performed partly under sponsorship of the U. S. Department of Energy ()flice of Arms Control and Office of Envirommental Restoration and Waste Management as part of a research and development program and dues not imply corrent or future policy decisions or technical preferences of any agency of the U. S. govermment.
} 
The neutron probe is based on a unique associated-particle sealed-tuhe neutron generator (APSTNG) that interrogates the object of interest with a low-intensity beam of $14-\mathrm{MeV}$ neutrons generated from the deuteriumtritium reaction and detects the alpha-particle associated with each neutron. Gamma-ray spectra of resulting neutron reactions identify nuclides associated with all major chemicals in explosives, drugs, and $\mathrm{CW}$ agents, as well as many pollutants and fissile and fertile SNM. Flight times determined from detection times of the gammarays and alpha-particles yield a separate low-resolution tomographic image of each nuclide identilied in the timecorrelated gammit-ray spectrum. By detecting only neutrons that have the proper flight-time to he uncollided, the APSTNG can also form the basis for a compact fast-neutron transmission imaging system with no collimator that can be used along with or instead of the emission imaging system.

In the following section, operational aspects of the system and its components are described, including detection modes and the sealed-tube neutron generator. In the next section, applications are discussed for interrogation of relatively small volumes, including treaty verification and related roles and detection of explosives and drugs. After that, systems are proposed for contraband detection in large cargo containers, including first and second stage emissive systems and a transmissive system. Finally, conclusions are drawn concerning APSTNG systems and applications, including strengths, limitations, and complementarity relative to other technologies and future development directions.

\section{OPERATIONAL ASPECTS OF SYSTEM AND COMPONENTS}

The neutron diagnostic probe which Argonne has been using was developed by the Advanced Systems Division of Nuclear Diagnostic Systems (NDS). ${ }^{2,3}$ Its operation can be understood from Fig. 1, which sketches a "general purpose" APSTNG system. The object to be interrogated might be baggage or a TLI (Treaty Limited Item: item to be inspected under arms control treaty provisions), that could contain explosives or drugs or CW or lissile material. In the APSTNG, deuterons are accelerated into a tritium target, producing 14-MeV neutrons isotropically. Each neutron is accompanied by an associated alpha-particle travelling in the opposite direction. The gamma-ray and neutron detectors are time-gated by pulses from the alpha detector, forming a cone of flighttime-correlated neutrons through the object. Detector pulses are time-resolved by CFD's (constant-fraction discriminators). Flight times are determined by a TAC (time-to-amplitude converter), digitized by an ADC (analog-to-digital converter), and recorded.

When a reaction occurs in the object along the cone that results in a detected gamma-ray, the time-delay from the alpha pulse yields the position (depth) along the cone where the reaction occurred, since the source neutron and gamma-ray speeds are known $(5 \mathrm{~cm} / \mathrm{ns}$ and $30 \mathrm{~cm} / \mathrm{ns}$, respectively). By scanning the alpha detector horizontally and vertically, or by using a two-dimensional (2D) position-sensitive multipixel alpha detector, transverse and depth coordinates of reaction sites can he mapped, providing three-dimensional (3D) emission imaging of reaction densities from measurements at a single orientation. In most applications, systems would probably include a 2D alpha detector, as well as an array of gamma-ray reaction detectors, so as to maximize infomation obtained from each interacting neutron and sufficienlly minimize measurement time.

Figure 2 illustrates the electronics and information flow for a basic multipixel system containing a 2D positionsensitive alpha detector and one gammit-ray reaction detector. In Fig. 2, the vectors involved in the reaction location are "Rt", "Rd", and "Rs". The transverse " $X "$ and " $Y$ " coordinates of "Rt" are digitized and stored in the PC computer in list mode, along with "TOF" (time-ol-flight = "t" - "T") and gammatray energy "Eg". Not shown in Fig. 2 is an alphat-gimma coincidence circuit that ensures the TAC receives only stant pulses that are followed by stop pulses. The PC controls the experiment, calculates positions, and displays data and images. Soltwate caur be developed for specific applications that will allow the PC to perform intelligent datat antalysis and interact with the operator to determine which items are sufficiently suspect to require further examination. 


\subsection{Detection modes}

Fast-ncutron inelastic scattering reactions in the object provide prompt gamma-ray spectrat that can identify many nuclides. By choosing gamma lines of specific nuclides, a 3D image of each identifiable nuclide in the time-correlated spectrum can he mapped. By choosing appropriate nuclide intensity ratios, 35) images of compounds can be made (molecular bonds are not identified). The use of the time-correlated gianma-raty spectra, as: discussed so far in Section 2, is denoted the EGRIS (emissive gamma-raly imaging and spectroscopy) mode. (If fissionable materials are present, neutron reaction detectors may be used to detect emitted lission neutrons). Nearly all nuclides with atomic number above horon have distinctive gamma-ray spectra for the EGRIS mode, with reaction cross sections of at least 0.5 barn or so for $14-\mathrm{MeV}$ neutrons (predominantly inelastic scattering reactions). For gamma-rays above $1 \mathrm{MeV}$ or so, background is greatly reduced since hackground counts call only be accumulated during the nanosecond-range correlation interval, yielding a high signal-to-background ratio. The EGRIS mode is generally the primary detection mode.

Slow-neutron capture is not time-correlated with the alpha pulses, but provides nonimaging gammatray spectra that can atd nuclide identilication. The use of non-correlated gamma-ray spectra with the neutron generator on is termed the CGRS (capture gammatray spectroscopy) mode. CGRS data cin be collected simultaneously with EGRIS data by using an MCA (multichannel analyzer) board inside the PC. The themnal neutron capture cross section is small for most nuclides, but is large for a few nuclides of interest in some applications. The gammatray spectra are generally substantially more complex than for EGRIS mode, with much more background. Often moderation provided by the interrogated object itself is ineffective. If extemal neutron moderation is used to get sufficient intensity, the EGRIS mode signal will be reduced due to depletion of fast neutrons.

Another detection mode of use is the PGRS (passive gamma-ray spectroseopy) mode, for which the neutron generator is turned off, allowing detection of gamma-ray radioactivity present in the interrogated object and in its. vicinity, including any activity induced in the object by the neutrons as well as uncorrelated background. This mode is used for static system energy calibration with gamma-ray sources (dynamic energy calibration is perfomed in the EGRIS mode using known materials and their reaction gamma-ray spectra). Because cross sections for inducing activity with a finite half-life (several seconds or more) are usually very small, the PGRS mode is generally not used with the APSTNG system for actual interrogation, unlesis natural giunmat-raty radioactivity is present (as for radioactive waste or nuclear weapons).

As shown in Fig. 1, by discarding detected neutrons not having the proper flight time to be uncollided, one can perform fast-ncutron 2D transmission imaging without a collimator (hy icanning, using a ncutron detector hodoscope array, or using 2D neutron detectors), since scattered neutrons are removed by "electronic collimation". This is called the FNTI (fast-neutron transmission imaging) mode. By measuring at a sufficient number of views around 180 degrees, 3D tomography is feasible. Transmission imaging (FNTI) can be done along with or instead of emissive reaction-density imaging (EGRIS). No spectral distinction hetween nuclides is provided, hut the neutron altenuation coeflicient is mapped over the interrogated object. (This mode is a method of perloming fastneutron transmission radiography, similar (o) x-ray radiography). The FNTI mode is used to map and position the

neutron correlation cone hut so far not heen much used for interrogation. But it maty find use in the future for simultaneous mode measurements, 10 correct EGRIS images for neutron allenuation or for multimode image analysis, or in a separate system for imaging extended or highly absorhing ohjects.

\subsection{Sealed-tube neutron generator}

The use of the associated-panticle method in arms control treaty verification and contraband detection research and development is recent. However the application of this method to NDA for neutron inelastic scattering is not new, although it has heen relatively undeveloped and confined to the lathoratory hecause of the bulk, complexity, and reliability and mintenance probiems of the accelerator equipment previously required. The replatement of 
the accelerator in this neutron diagnostic probe system by the sealed-tube APSTNG brings new flexibility to the method and allows it to become a tool for field use. The state-of-the-art APSTNG wats developed by the Advanced Systems Division of NDS, after considerable experience.

As diagrammed in Fig. 3, a Penning ion source inside the APSTNG emits a continuous mixed hean of deuterium and tritium ions that is accelerated and focused on a small spot $(\sim 1 \mathrm{~mm}$ diameter $)$ on the target, tritiating the target and producing neutrons and alpha particles. (The small spot is necessary to obtain good spattial resolution.) A zirconia getter controls the mixture of deuterium and tritium. The ions are accelerated hy a high voltage of $95 \mathrm{kV}$ and focused by a variable extraction voltage, nominally $15 \mathrm{kV}$. The ion heam current $(\sim 1 \mu \mathrm{A}$ to get $\left.10^{6} \mathrm{n} / \mathrm{s}\right)$ is controlled by varying the getter heating current. All operating voltages and currents are furnished and monitored by a high-voltage control system. Internal pressure is $\sim 10^{-5}$ Torr without getter heating and $\sim 10^{-5}$ Torr under normal operating conditions. The welded metal-ceramic tube contains ().4 Ci tritium at low pressure compared to the atmosphere, and the tritium is contained in the getter when the tuhe is not operating. A $1.6 \mathrm{~mm}$ lead shield reduces operator exposure $10 x$-rays from the ion source.

The single-pixel alpha detector consists of a $\mathrm{ZnS}$ screen inside the tube, with a photomultiplier cutside interfaced to a window. (In the case of a multipixel 2D alpha detector, the photomultiplier can be replaced by a microchannel plate and resistive anode readout, as shown in Fig. 3.) The alpha scintillator is limited to materials that can withstand tube bake-out temperatures during manufacture. $\mathrm{ZnS}$ is fast enough, has high light output, and is inexpensive.

The operating history of each APSTNG made by NDS has been maintained. Initial maximum output of a typical APSTNG is around $3 \times 10^{7} \mathrm{n} / \mathrm{s}$, but the maximum output soon falls to about the level of $10^{7} \mathrm{n} / \mathrm{s}$, as the cathode target is sputtered away, and slowly decreases thereafter. An output off $\sim 10^{6} \mathrm{n} / \mathrm{s}$ can be maintained for $\sim 2000$ hours by increasing the ion current to compensate for sputtering. Eighteen ceramic tubes have hecn made, fourteen of which passed quality control test: (a 78\% yield). Eleven of these tubes were put in operation (three are in stock), of which six are still operating. Three tubes failed in nomal operation; two were failed by continuous operation at excessive voltage. For the nine tubes which were operated nomally, the average lifetime output is $7.35 \times 10^{12}$ neutrons, if end of life is defined as being reached when the maximum output hecomes less than $10^{6} \mathrm{n} / \mathrm{s}$.

The design of the APSTNG differs substantially from the current well-logging neutron generator tubes, which cannot be used for associated-particle operation. (Well-logging tuhes are usually pulsed, have no capability to focus the ion bean on a small spot on the target, and have no internal scintillator.) The APSTNG is ant inexpensive small sealed module with low-hulk support equipment. It has a long mean-lime-between-failures (around $20(0)$ hours at $10^{6} \mathrm{n} / \mathrm{s}$ or $2(0)$ hours at $10^{7} \mathrm{n} / \mathrm{s}$ ), is easily replaced (allowing simple field operation), presents low ratdiation exposure, and the sealed-tuhe design prevents tritium contamination.

\section{APPLICATIONS FOR INTERROGATION (OF SMALL VOLUMES}

The APSTNG primary EGRIS detection mode is optimal for (small) volumes of dimension $\sim 1$ m on al side or less, for objects that do not strongly ahsorh 14-MeV neutrons or gammat-rays above $1 \mathrm{MeV}$. APSTNG technology then has the capabilities for identification and 3D imaging of many individual nuclides and componunds, with flexible positioning of reatetion detectors with respect to the neutron soure (on the same side, perpendicular, or opposite side), as well as capability for fast-neutron transmission imaging. The source and enitted radiation are high-energy and penetrate dense objects. Proof-of-concept lahoratory experiments have heen suecessfully done for a number of applications: nuclear warhead detection, chemical ordnance identification, explosive detection and identification, contrahand drug detection, uranium borehole logging, corrodent detection (nn stean-turhine hlades, kerogen antalysis of shale, on-line assay of coats, and hulk soil remediatton of radwaste and pollutants. 


\subsection{Treaty verification and related roles}

Many of the general requirements for a detection system in treaty verification roles for on-site inspection are similar to those for contraband detection, such as an unambiguous decention-resistant signal (to minimize false positives and negatives), maximum performance to cost ratio (10 minimize fielding costs), easy operation and maintenance (not requiring highly trained personnel), and portability or transportability (for remote field use). However additional operational and equipment constraints are imposed by the treaty and its ranifications, such as protocols denoting sites and conditions of on-site inspections, rules for counting and classifying weapons, desire of the host country to reduce site disruption and to minimize intelligence and weapon design/manufacture information obtained, and desire of the country that developed the system to minimize technology transfer. Often the same detection system will be required to be used by all parties to a treaty. The equipment maly he restricted in energy and spatial resolution and in technologies used, should be as simple and as transparent as possible in function and operation, and may have to operate in various extreme climates.

Proof-of-concept experiments on nuclear warhead detection and counting have heen performed in both the EGRIS and FNTI modes on crude mockups containing simulant materials. ${ }^{5,6}$ In the EGRIS mode, the APSTNG system was able to separately identify and image explosive and SNM simulants with sufficient energy and spatial resolution to identify and count nuclear warheads on missiles, despite substantial attenuation. In the FNTI mode, sufficient penetration yet adequate contrast and sufficient spatial resolution were demonstrated, to allow counting of nuclear warheads using a multiview tomographic technique. New experiments on simulant mockups of individual nuclear weapons may lead to the capability to identify components, to distinguish simulated weapons from real ones, and to nonproliferation roles.

Early investigations of APSTNG detection capability for CW agents and munitions were promising, including an analytic study of gamma-ray energies and cross-sections for neutron inelastic scattering and element structure of $\mathrm{CW}$ agents and explosives, as well as measurements taken of $155-\mathrm{mm}$ shells filled with $\mathrm{CW}$ simulants using

an early version of the APSTNG system. ${ }^{5,6}$ Recently we have measured spectra of the basic elements involved as well as of new accurate CW surrogates we have prepared for some nerve and mustard agents, using our new large double-ended Nal detector. Our results indicate that an HPGe detector, with its high energy resolution, may be needed to supplement our Nal detector for identification of agents having overlapping closely spaced lines in their gamma-ray spectra. Experiments are planned using an HPGe detector for CW munition identification, that may lead to other applications involving complex spectra, such as remediation of radioactive or toxic waste. As illustrated in Fig. 4, simultaneous identification and imaging of individual munitions in cartridge boxes and pallets, without requiring opening and breaking out of the rounds for separate measurements, is a potential EGRIS-mode advantage in $\mathrm{CW}$ detection.

\subsection{Detection of explosives and drugs}

Based on data from APSTNG laboratory experiments on explosives and drugs, it is interesting to estimate the measurement times required for detection of contraband items in some simple idealized cases using a lielded APSTNG system, in this case, one having a multipixel alphatparticle detector and eighteen relatively lange douhleended Nal gammit-ray detectors, as shown in Fig. 5 (see Sec. 5.2 for an explanation of the double-ended Nal detector). The lathoratory data consists of EGRIS gammat-raty spectrat for nitrogen, oxygen, and carbon, for specific explosives (C-4, PBX, Octol, Composition B, TNT, Pentolite, Dyntmite, and Dela sheet), and for cocaine and heroin simulints.

The spectrum for a typical explosive, such at C-4 shown in Fig. 6, exhihils a number of principal peaks (mosily from neutron inclastic scallering) characteristic of nitrogen, oxygen, and carthon, alkng will at number of escape peaks, with a number of peaks overlapping. (In Fig. 6, "SE" and "DE" refer to single and double escape peaks, respectively.) The proportion of nitrogen is relatively high for conventional explosives, compared (1) olher 
materials likely to be present (the density of explosives tends to be high also). The spectrum for a typical narcotic, such as cocaine or heroin, shown in Figs. 7 and 8, tends to have a high proportion of carbon compared to oxygen. Other materials that might he present in shipments containing contraband drugs include sugar and halite, whose spectra are given in Figs. 9 and 10. Sugar has a much lower proportion of carbon to oxygen than cocaine or heroin, and halite contains no carbon or oxygen.

By a judicious choice of energy windows and knowledge of the hasic nitrogen, oxygen, and carbon spectra, a "detection" algorithm was obtained that correctly reproduces the density ratios of nitrogen to oxygen and carbon 10 oxygen for the explosives and for other materials that might he present. It was found that a plot of these ratios is useful for differentiating contrahand drugs or explosives from ordinary items expected to be seen (see Fig. 11). Materials that would normally appear in luggage or foodstuffs are separated from explosives (open squares) and cocaine.

The hoxes shown in Fig. 11 represent count statistics for a 15 -second measurement of $1 \mathrm{~kg} \mathrm{C-4} \mathrm{explosive,} \mathrm{the}$ larger box enveloping five standard deviations (5 sigma), the smaller box enveloping two standard deviations. (The C-4 is assumed to be located in one volume element, rather than being spread out in a sheet.) The C-4 is definitely identified as an explosive. In fact, even a 4-second measurement would distinguish the C-4 from items normally found in luggage, so if luggage is being examined, suspicion is indicated. In 480 seconds of measurement time, the $\mathrm{C}-4$ would be differentiated from other common explosives.

In Fig. 12, boxes are drawn for two and five standard deviations of count statistics for a 2-second measurement of $1 \mathrm{~kg}$ of meat. With high probability, the meat is identified as a foodstuff rather than explosives or contraband, indicating a very low false alarm rate for monitoring foodstuffs. In Fig. 13, a Iwo-standard-deviation box is shown for a 4-second measurement of $1 \mathrm{~kg}$ of cocaine in fish. (The cocaine is assumed to occupy only one volume element.) The item is identified as suspicious, since it is not just fish, and is thought probably to be cocaine, since it is not amphetamines (it could be plastic, but why would plastic appear in fish?).

These simple computations and the underlying laboratory data can be considered as comprising a feasibility study for the application of an APSTNG system to monitoring contraband explosives and drugs, but are of course no substitute for analysis of laboratory measurement of realistic mockups and actual field trials of prototype units. The presence of noncontraband materials (attenuation and scattering effects and the complex arrangements of many diverse items that may normally appear) and deception attempts (such as explosives and drugs in sheet form, shielding, judicious placement of noncontraband materials, and adulteration of drugs) have not heen considered. On the other hand, much more powerful detection algorithms that utilize material densities directly along with full spectral regression fitting, adaptive learning processes, and extemal information from other surveillance methods call he developed.

\section{CONTRABANI) DETECTION FOR CAR(GO CONTAINERS}

The criteria for setting required detectable anounts of contraband and meatsurement limes are yuite different for cargo entainer and truck inspection than for aviation security, since for trucks or containers, false negatives are not immediately catastrophic for small amounts of contraband that are hard to detect and false pesitives do not greatly affect very many people immediately. A (wo-stage inspection anangement in which the first slage searches for "suspicious" (argo over the full container and a second stage identifies contraband rather precisely for oftloaded suspicious cargo should he cost-effective in recovering most of the contraband and in reducing labor in offloading/reloading and unpacking/packing caused hy false alarms. 


\subsection{First and second stage ECiRIS systems}

A single small EGRIS system is not very suitable for imaging extended objects much over a meter or so) in all three dimensions or highly absorbing objects. For large objects, the double solid angle reduction (source-10ohject and ohject-1(1-detector) substantially reduces signal count rate. Accidental counts hecome limiting, and the raw images exhibit amplitude reductions and increased fluctuations in regions where neutron or gammatray allenuation are significant and increased background and distortion where scalliering is substantial.

As the first stage in examination of a truck or cargo container, a gantry system of multiple EGRIS stations is proposed, ${ }^{7}$ as shown in Fig. 14, a schematic representation of the end view (circled numbers associate each APSTNG with its detector array). Each of the four stations is located at a different position along the drive path, so that each station interrogates the full length. The arrangement is designed to cover the full cross-section of the cargo container (24.4 $\mathrm{m}$ on a side), concentrating as much as possible on the center region, where detection sensitivity is worst, while reducing distances between neutron generators, reactions in the container volume, and detectors to improve overall sensitivity. A simple calculation based on differentiating cocaine from polyurethane in Fig. 12 and on average neutron and gamma-ray attenuations of $0.0165 \mathrm{~cm}^{-1}$ for normal cargo $\left(\sim 0.5 \mathrm{~g} / \mathrm{cm}^{3}\right.$ average density) indicates that a 12.2-m long cargo container can be inspected in less than 30 minutes, for 12 large double-ended Nal detectors per station, with a sensitivity ranging from $\sim 0.5 \mathrm{~kg}$ cocaine near the walls $10 \sim 20$ $\mathrm{kg}$ cocaine near the center. (This calculation does not consider deception attempts or the wide variety of possible arrangements of diverse items that may be present). This stage of inspection should substantially reduce false alarms that unnecessarily result in the heavy labor of offloading cargo containers.

A second stage of examination is proposed consisting of the relatively small EGRIS unit shown in Fig. 5. This stage should he able to positively identify drugs and other contrabiund within $\sim 0.5 \mathrm{~kg}$ or hetter in several minutes or less of measurement time, for items less than a cubic meter in volume, and would interrogate cargo that is offloaded hecause it has heen identified as suspect by the preceding stage or is $1(0)$ well shiclded to be interrogated by other stages. By reducing false alarms and locating contraband more precisely, this stage should signiliciantly reduce the substantial labor of unpacking and repacking offloaded cargo.

\subsection{FNTI and (GR'TI systems}

Unlike in the EGRIS mode, in the FNTI mode the signal is proportional to the source-to-detector solid angle alone, and scattering is also less of a problem. An FNTI system would hetter penetrate more high absorbing cargo containers and is proposed as the first stage inspection system for cargo more dense than $\left(0.5 \mathrm{~g} / \mathrm{cm}^{3}\right.$ when averaged over the container, rather than the above first-stage EGRIS system. The end view of an APSTNG FNTI station with a transmisision hodoscope array of (fast-plastic) neutron detectors suitahle for imaging a cargo container or truck is shown schematically in Fig. 15. Fan-beam overlap aids container-center sensitivity. In addition to the iwo stations shown, anther series of Iwo stations having an overlap region rotitled 9() degrees would he imposed along the drive path, 10 linther improve sensitivity in the container center. Cross-section images over the length of the container or truck are obtained hy driving through the four stations. Other stations with different view angles may he installed allong the driving path. The actual number of stations and number (size) of detectors per station depend on the view angles and spatial resolution needed for detection of a specific amount of contrahand in a prescribed measurement time. If true tomographic imaging is required, the hodoscopes can be rotated.

As at stand-allone system, the FNTI hodoscope can only detect spatial allenuation antomalies (that maty have other causes than contrahand), in common with other typical transmission systems. We propose fo supplement it with a companion gamma-ray transmission imaging hodoscope (GR'Tl) system of stations similar lo Fig. 15, will the APSTNG replaced hy a small isotopic gammat-ray source (possibly $20 \mathrm{mC \textrm {Ci }}$ of ${ }^{611} \mathrm{Co},{ }^{1.37} \mathrm{Cs}$, or ${ }^{2.32} \mathrm{U}$ ) and the neutron detectors replaced hy gammatray detectors. The GRTl and FN'Tl systems are complementary, in that the gammatrays are altenuated more strongly by hign-Z materials, with $\sim Z^{2}$ dependence, and the neutrons are 
attenuated more strongly hy low- $\mathrm{Z}$, with $\sim(\mathrm{Z}+\mathrm{N})^{1 / 3}$ dependence $(\mathrm{Z}$ is the atomic number and $\mathrm{N}$ is the number of neutrons in the nucleus). Pixel-by-pixel comparison of the images ohtained promises to identify contraband drugs and explosives hidden inside high- $Z$ material or even hidden inside low-Z material, by observing spatial anomalies calused by differences in average $Z$ and density hetween contraband and other materials. If necessary, tomographic imaging would allow complete mapping of density and (material-averaged) $Z$ over the interrogated volume.

\section{CONCLUSIONS}

The proof-of-concept experiments for nuclear warhead detection show a potential for high-confidence measurements in treaty verification applications for detection, counting, and dismantlement of nuclear weapons. The proof-of-concept experiments for $\mathrm{CW}$ agents indicate a means other than chemical sampling is available and should be effective for verification of these agents, a method that requires no opening of sealed containers, penetrates intervening materials, and is nonintrusive regarding sensitive molecular hond information. The treaty verification instrumentation criteria of transportability, reliability, easy operation and maintenance, personnel salfety, and relatively low cost, would be met in these applications. Intrusion can be limited by limiting allowed output, measurement time, or time, space, or energy resolution, or by encryption of data.

Combining hodoscope technology with APSTNG technology opens avenues to a wide range of applications, not only for treaty verification, but also for nonproliferation surveillance of weapon materials, detection of contraband drugs and explosives, and remediation of radwaste and pollutants. A feasibility study indicates strong potential for success in detecting contraband explosives and drugs with APSTNG technology, with scalability from small systems for suitcase inspection to large multi-stage systems for cargo container inspection. However much remains to he done before any applications can be fully realized. For each specific application, realistic laboratory mockup experiments, development of deception-resistant detection algorithms, and fabrication and field testing of prototypes will be required.

\subsection{Strengths, limitations, and complementarity}

Relatively small and inexpensive but reliable and easily maintained APSTNG systems can identify and separately image most elements having a larger atomic number than boron. Chemical substances are identified and imaged by comparing relative spectral line intensities with ratios of elements in reference compounds. The high-energy neutrons and gamma-rays will penetrate large objects and dense materials. The source and emission detection systems can be located on the same side of the interrogated volume, allowing measurements with access to one side only. A high signal-to-background ratio is obtained and maximum information is extracted from each detected gamma-ray, yet high-bandwidth data acquisition is not needed. No collimators for the source or detectors are required, and only minimal personnel radiation shielding is needed. A single-prohe system can simultaneously interrogate all volume elements of an object up to cubic meter or so in size (no scanning is required).

There are some limitations in the EGRIS mode of the present APSTNG system that can he significant for many applications, even for interrogation of volumes that are not large. Presently attainable depth resolution is limited, for a small gamma-ray detector, 10 ahout $5 \mathrm{~cm}$ (hecause the system has an overall time resolution of $\sim 1$ ns and a 14-MeV neutron travels $5 \mathrm{~cm}$ in I ns), and measurement times can be lengthy to ohtain sufficient gammat-raty counts from a single gammatray detector. The gammatray signal count rate is limited hy reaction cross-sections, solid angles subended by the alpha detector and gamma-ray detectors, gamma-ray detector efficiency, and neutrom source strengh, but usible source strength is limited hy accidental counts and pileup. However a gimmma-raty hodescone detector array can he used to substantially increase the signal count rate to lower meatsurement times, when necessiary.

A pulsed fast-neutron system hased on similar principles as the APSTNG (obtaining depth resolution using flight times) has heen proposed for cargo container inspection. ${ }^{8}$ Because of the requirements of strong source and 
detector collimation, scanning, and nanosecond pulse width, a bulky complex high-intensity accelerator with heavy shielding is needed that is expensive to develop and maintain, instead of the few easily replaced tuhes in the proposed APSTNG gantry EGRIS system. The proposed FNTI/GRTI system provides a lower-cost lower-hulk more easily maintained altemative (1) an intense high-energy x-ray system (with its complex alceclerator and heavy shiclding) for inspecting densely loaded cargo containers;" the FNTI/GRTI system will have lower spattial resolution but hetter atomic-number specificity.

It has been pointed out that more effective surveillance of explosives in the aviation security environment would result from integration of different types of sensors and detection methods in a sequential and/or logic arrangement. ${ }^{10}$ Another option is to directly use complementary information in an integrated fashion to maximize detection probability while minimizing both false-alarm rates and inspection times, rather than using sequential measurement, which can tend 10 minimize false-alarm rates at the expense of lowered detection probahility (or vice-versa). For the aviation security environment and other surveillance applications, the vast majority of objects interrogated will not contain items being screened for, so the occurrences of both false positives and false angatives should be minimized. The complementary systems approach is a means of accomplishing this goal.

For example, x-ray data, which can distinguish density differences and provide fine spatial detail, is complementary to APSTNG EGRIS data, which provides relatively coarse spatial resolution but is highly specific to explosives materials. The $x$-ray data can be used to define suspicious shapes and densities that might indicate explosives or detonators (particularly if the $x$-ray unit is tomographic or dual-energy). This data would he passed on to the APSTNG unit, which would measure only the suspect volume, at the most advantageous orientation for detection as determined from the $x$-ray data, reducing the measurement time required by the APSTNG unit for positive identification. A computer would integrate the data from both units for final determination. The complementary $x$-ray/APSTNG system could be fine-luned for the desired high detection prohahility and low falsealarm rate. As another example of complementari!y, the proposed second-stage EGRIS unit for cargo container inspection could be used simultaneously with a first-stage unit for maneuverable inspection of appendages not easily viewed by the first-stage unit, such as tires, franes, and truck cabs.

\subsection{Development directions}

In developing gamma-ray hodoscope detector arrays, one must understand that small detectors will have at simall energy-peak efficiency for the high-energy gamma-rays detected, while large detectors of the standard form will degrade the time resolution (and thus the depth resolution) substantially. Detector configurations are being investigated that will substantially improve the signal count statistics, while retaining ats much time and energy resolution as possible. One configuration being investigated is a long Nal detertor with two photomultipliers, one at each end; improvements in time and energy resolution are heing ohtained by combining the timing and energy data from the two ends. Testing of HPGe detectors for integration into the detector arraty for help with antalysis of complex spectra will take place soon. Another development need is hardware and software configurations for specific applications, including miniaturization and ruggedization of components for transpontable applications.

For most applications (including physical and aviation security), the usiable neutron source strength is expected to he limited to the range of $10^{6} 10100^{7} \mathrm{n} / \mathrm{s}$ for the associated particle method, for which the present APSTNG tule hats adequate outpul and lifetime. Although there is some incentive to develop an APSTNG tuhe with higher output and longer lifetime for some applications, the development may he difficult and uncertain. A fassier, less expensive, and more certain development path is 10 increatse the number of gammatray detectors. Even a latrge array of detectors can be made lo he fairly compact and lo operate reliahly, with little maintentule required. 'In this way, the present APSTNG, which exhihits high reliahility and can he quickly replated, cann he used in mally applications. Surveillance systems hased on APSTNG technology ciun avoid the large physical size, high calpitil and operating expenses, and reliability problems associated with complex accelerators. 


\section{ACKNOWLED(IMENTS}

The support and direction of M. F. O'Connell, Project Manager for the Office of Ams Control, U. S. Deparment of Energy are greatly appreciated. We acknowledge, with many thanks, the cooperation and support of the NDS staff, A. H. Aitken, C. C. Blackmon, and C. J. Henderson. We also appreciate the continuing direction and support provided hy Argonne Arms Control Progran Maulager A. Travelli and hy A. DeVolpi.

\section{REFERENCES}

1. A. DeVolpi, C. L. Fink, G. E. Marsh, E. A. Rhodes, and G. S. Stanford, "Fast-Neutron Hodoscope at TREAT: Methods for Quantitative Determination of Fuel Dispersal", Nucl. Tech., vol. 56, pp. 141-188, Jan. 1982.

2. C. M. Gordon and C. W. Peters, "A Fast-neutron Probe for Tomography and Bulk Analysis", Int. J. Radiat. Appl. Instrum. Purt A, vol. 41, pp. 1111-1116, 1990.

3. C. M. Gordon and C. W. Peters, "A Fasi-neutron Probe for Tomography and Bulk Analysis", Trans. Amer. Nucl. ioc', vol. 56 (Suppl. 3), pp. 1-86, 1988.

4. C. M. Gord on and C. W. Peters, "Instrumental Assay of Kerogen in Shade Using Fast Neutrons", Imt. J. Radiat. Appl. Instrum. Purt E, vol. 2, pp. 123-128, 1988.

5. E. Rhodes, C. E. Dickerman, A. DeVolpi, and C. W. Peters, "APSTNG: Radiation Interrogation for Verification of Chemical and Nuclear Wcapons", IEEE Trans. Nuci. Science, vol. 39, pp. 1041-1045, Aug. 1992.

6. E. Rhodes and C. W. Peters, "APSTNG: Neutron Interrogation for Detection of Explosives and Drugs and Nuclear and CW Materials", SPIE Proceedings: Neutrons, X Rays, and Gamma Rays: Imaging Detectors, Material Characterization Techniques, and Applications (21-22 July 1992, San Diego, California), vol. 1737. pp. 1611-17(), 1993.

7. E. Rhodes and C. W. Peters, "APSTNG: Neutron Interrogation for Detection of Drugs and other Contral:and", Proc. Contraband and Cargo Inspection Technology International Symposium (28-30 Oc:t. 1992. Ommi Shoreham Hotel. Washington, D. C.). pp. 37-44.

8. Douglas R. Brown, Robert Loveman, Joseph Bendahan, and Martin Schulze, "Cargo Inspection System Based on Pulsed Fast Neutron Analysis", ibid. Ref. 7 above, pp. 235-241.

9. Rohert D. Rathmell, Jaunes B. Schroeder, Mark L. Sundquist, James R. Adney, and James E. Raatz, "Peilletron Accelerators for PFNA and MeV X-Ray Applications", ibid. Ref. 7 above, pp. 455-460).

10. A. Faunherg, "Explosives Detection for Aviation Security", Science, vol. 25.5, pp. 1531-1537, 20 March 1992. 


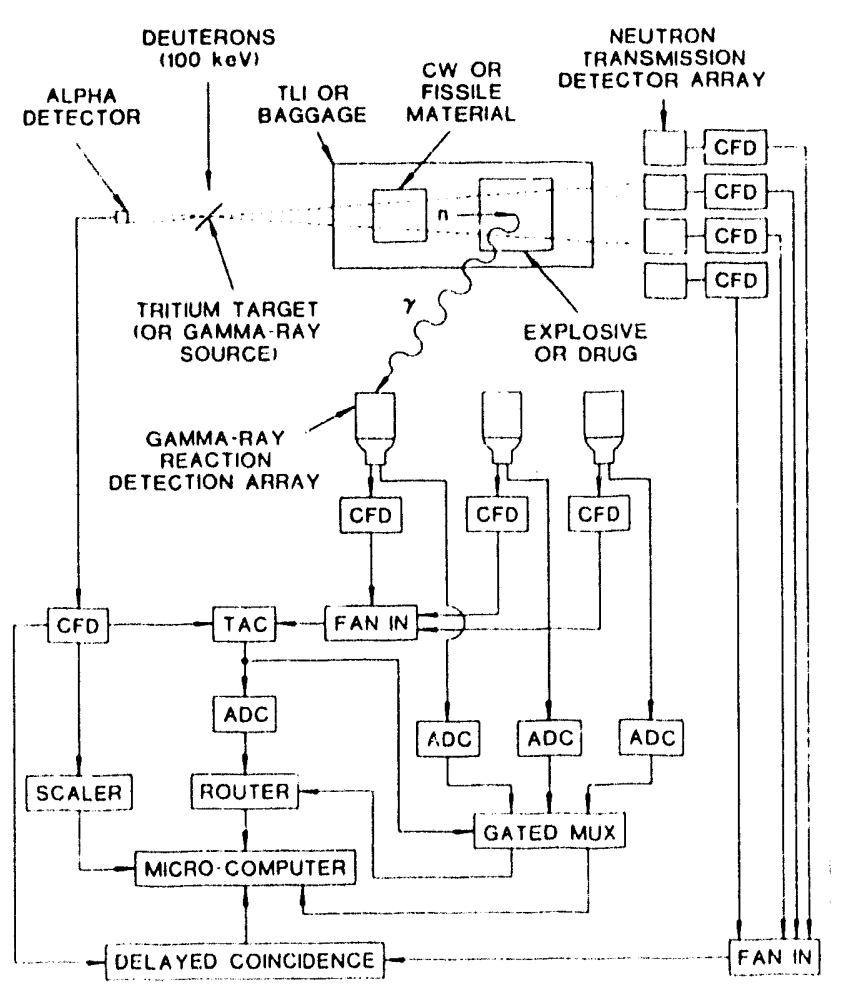

Fig. 1. Schematic layout of "general purpose" APSTNGbased interrogation system, with EGRIS and FNTI modes.

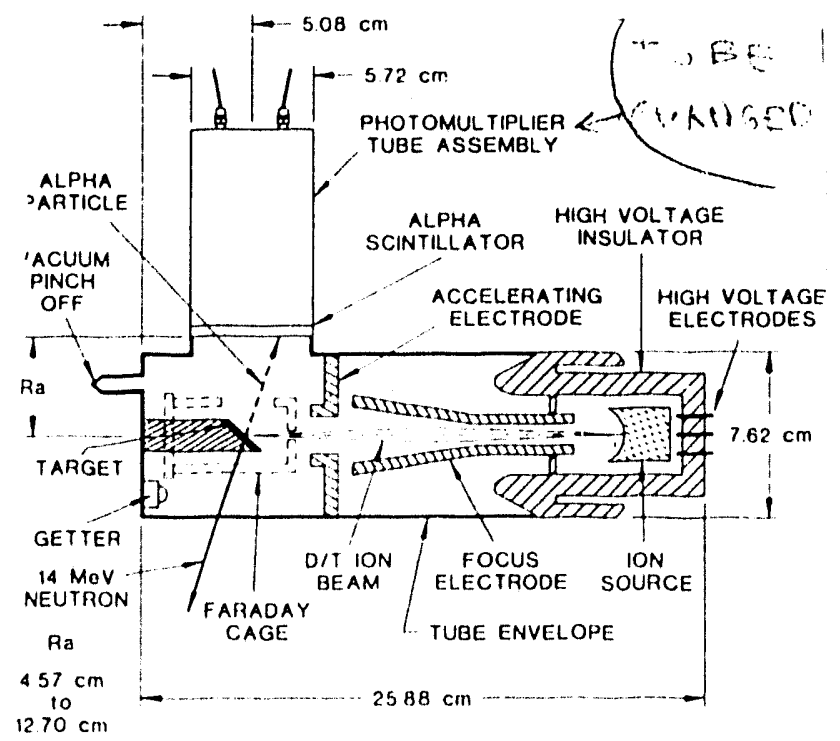

Fig. 3. Cross-section of interior of APSTNG tube, with 2D position-sensitive multipixel alpha-particle detector.

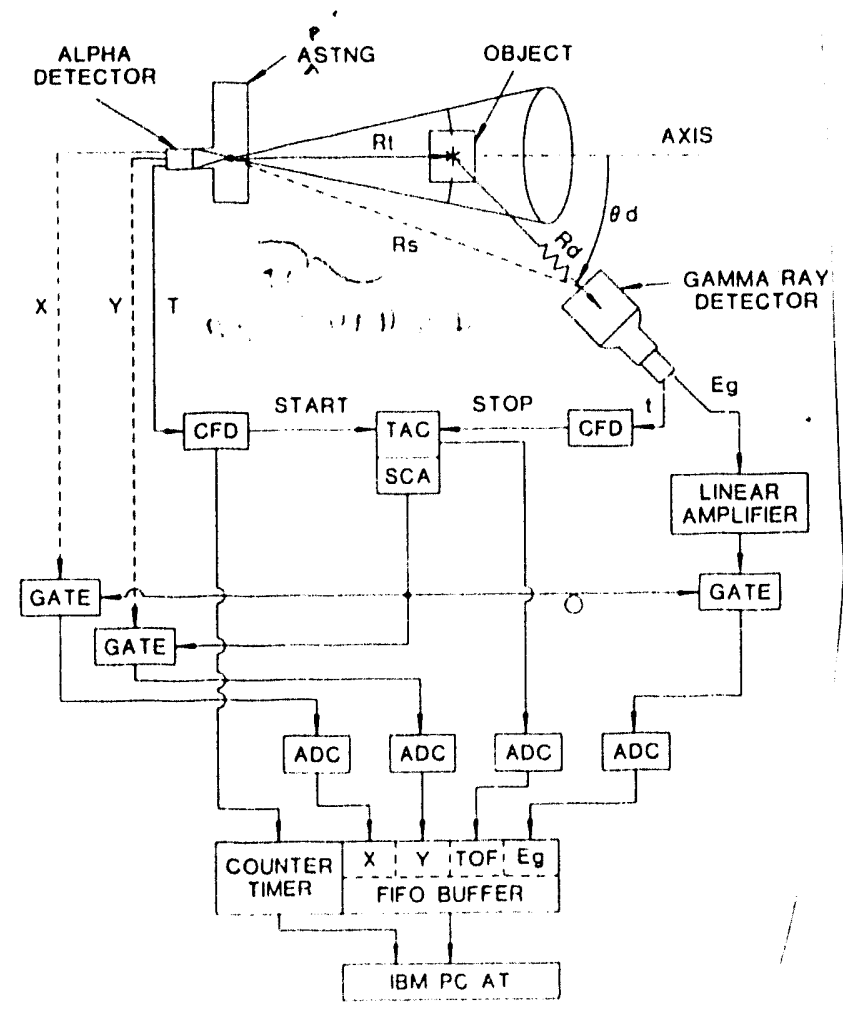

Fig. 2. Electronics and information flow for EGRIS-mode multipixel APSTNG-based system with 2D position-sensitive alpha-particle detector and one gaunma-ray detector.

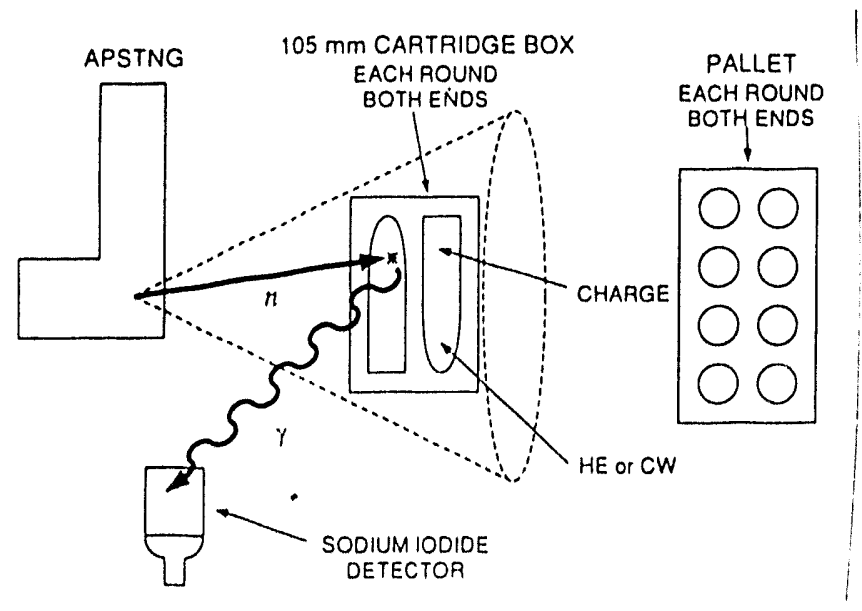

Fig. 4. Identification and imaging of $\mathrm{CW}$ munitions with transportable APSTNG system. 


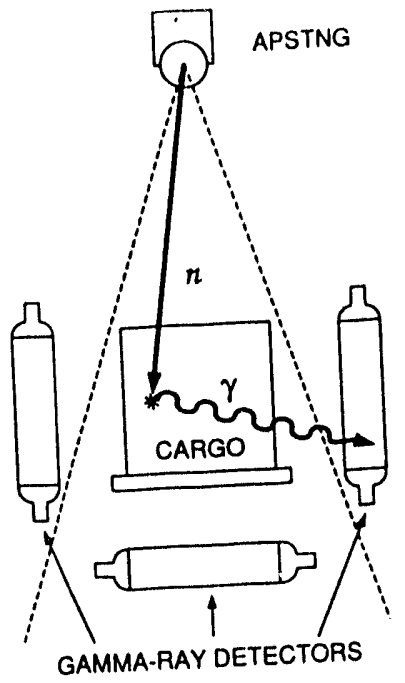

Fig. 5. Design concept of small-volume EGRIS system, end view. Five more detectors are behind each double-ended gamma-ray detector shown (PM tube at each end), a total of $18 \mathrm{NaI}$ detectors, each $15 \mathrm{~cm} \times 15 \mathrm{~cm} \times 45 \mathrm{~cm}$.

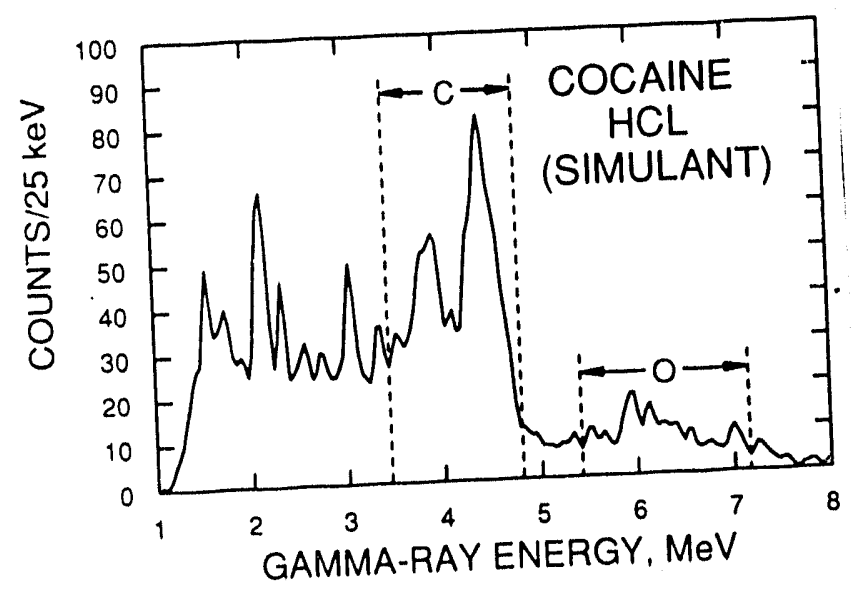

Fig. 7. EGRIS spectrum of cocaine $\mathrm{HCl}$ simulant.

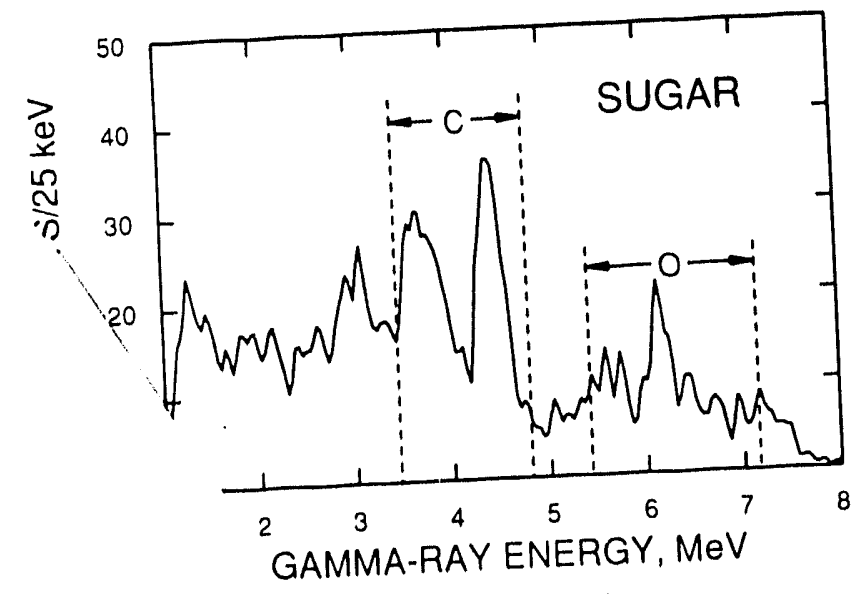

Fig. 9. EGRIS gammatray spectrum of sugar.

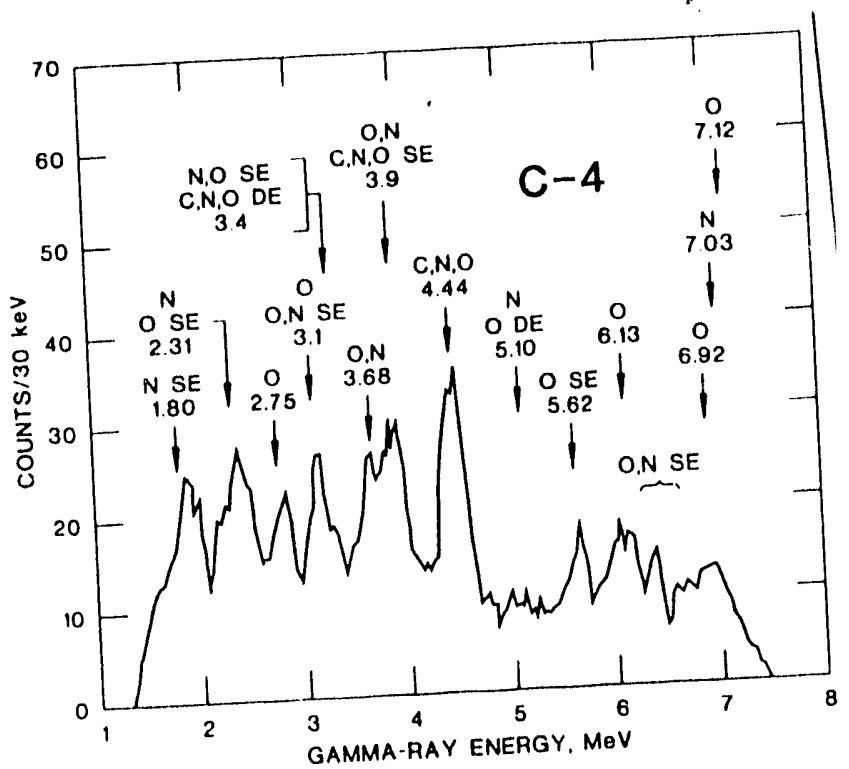

Fig. 6. EGRIS gamma-ray spectrum of $\mathrm{C}-4$ explosive.

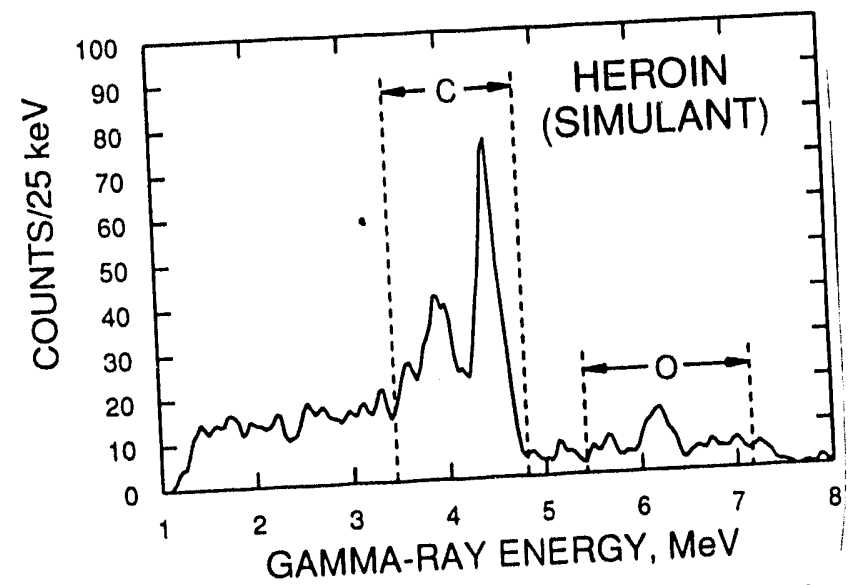

Fig. 8. EGRIS gamma-ray spectrum of heroin simulant.

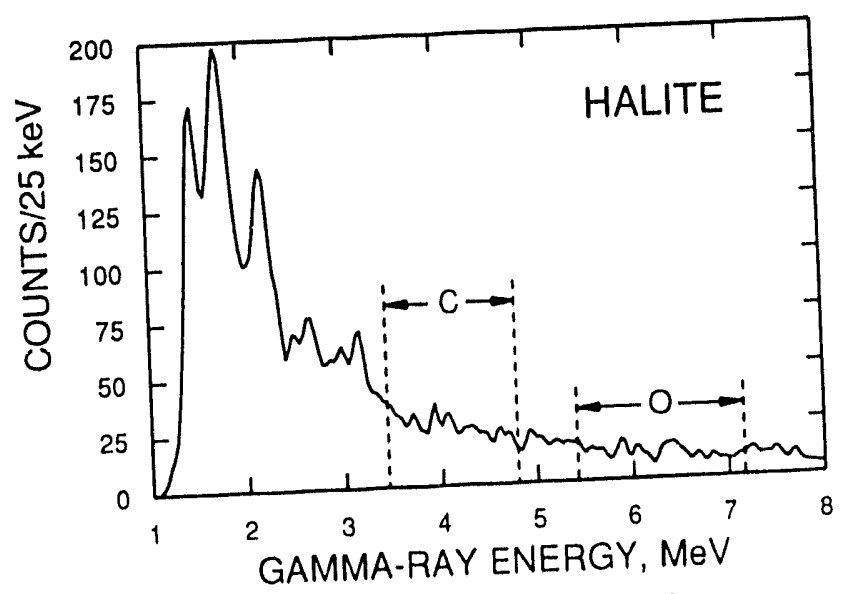

Fig. 10. EGRIS gamma-ray spectrum of halite. 


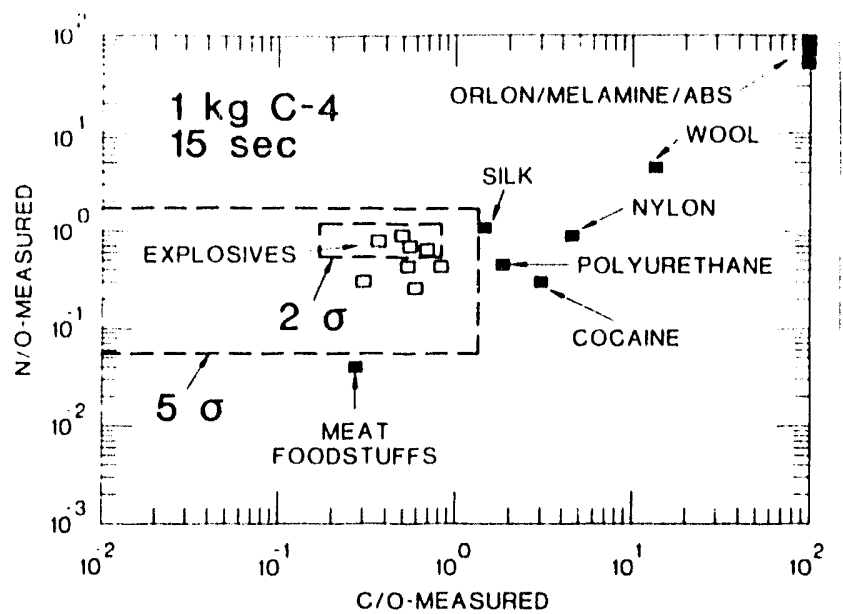

Fig. 11. N/O vs. C/O for 15 -s measurement of $1 \mathrm{~kg} \mathrm{C}-4$. Large box envelops 5- $\sigma$ statistics; small box envelops 2- - .

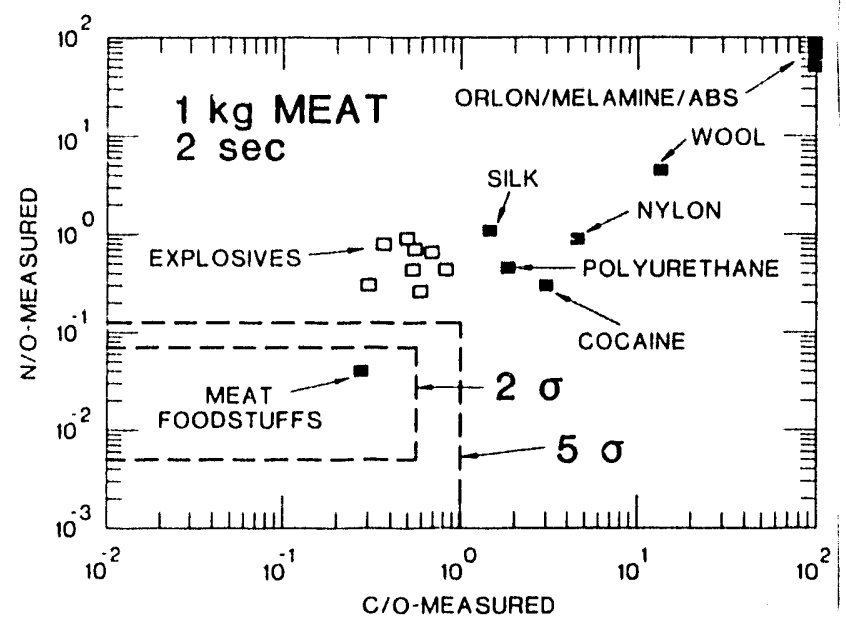

Fig. 12. N/O vs. C/O for $2-\mathrm{s}$ measurement of $1 \mathrm{~kg}$ of meat. Large box envelops 5- $\sigma$ statistics; small box envelops 2- $\sigma$.

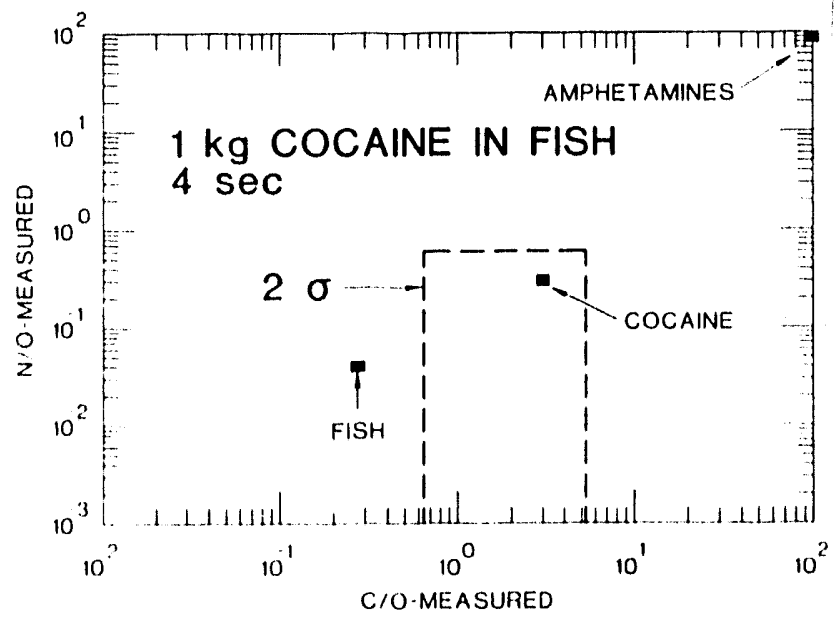

Fig. 13. APSTNG N/O vs. C/O for $4-\mathrm{s}$ measurement of $1 \mathrm{~kg}$ of cocaine in tish. Box envelops $2-\sigma$ statistics.

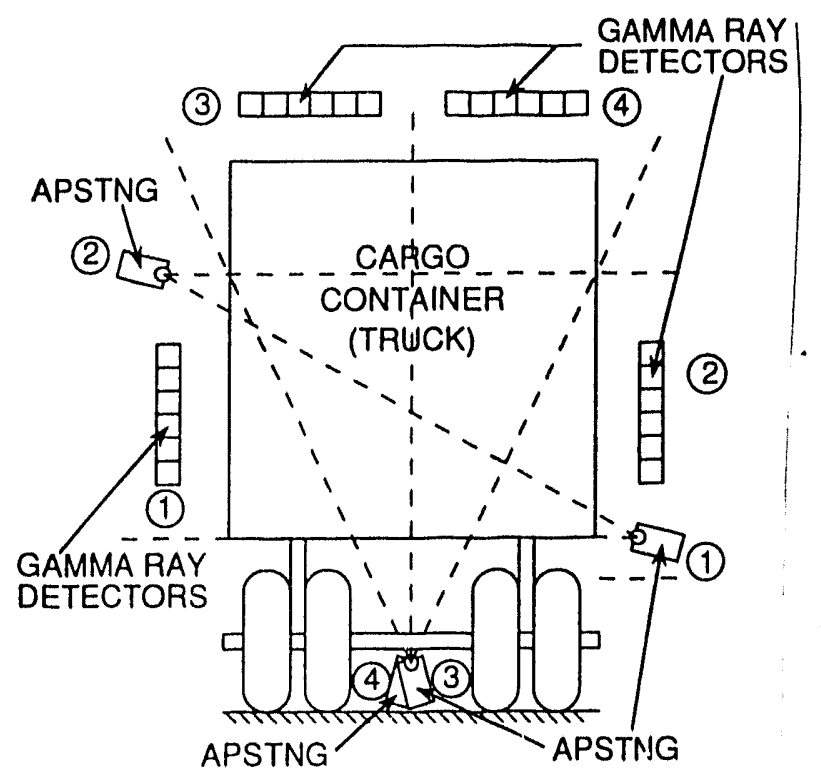

Fig. 14. Design concept of first-stage EGRIS system, end view. The four APSTNG-detector assemblies are located at different points along the drive path. Another detector array is located behind each 6-detector array, a total of 48 doubleended $15 \mathrm{~cm} \times 15 \mathrm{~cm} \times 45 \mathrm{~cm}$ Nal detectors.

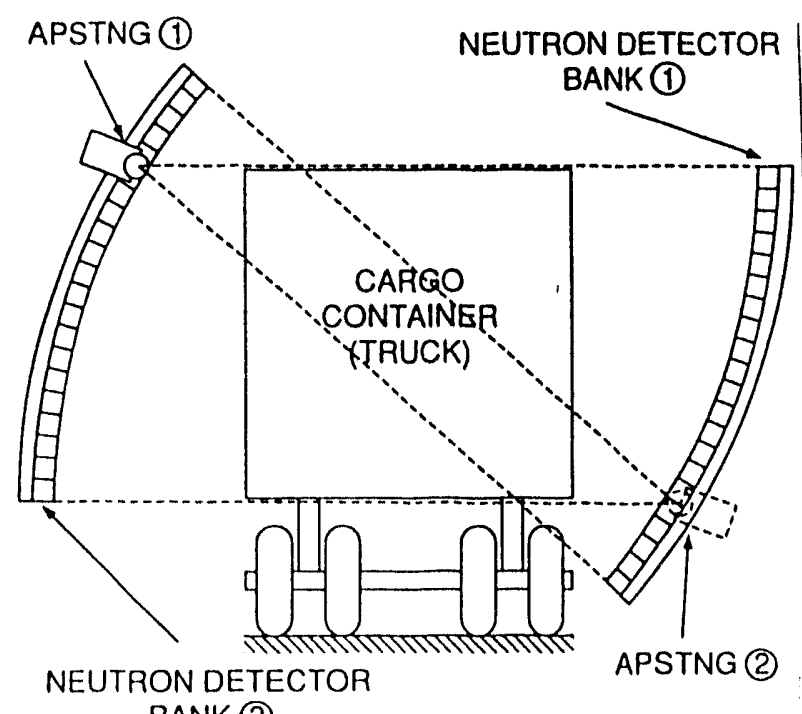

Fig. 15. Design concept of FNTI system, end view. The two APSTNG-delector atssemblies are located at different points along drive path. Four more banks of detectors are located behind each 21-detector hank shown, a total of 210 cubic $1.5-\mathrm{cm}$ plastic neutron detectors. 

